Botanical record club.

The London catalogue of British mosses. Comp. ...by C.P.Habkirk and H.Boswell. [ 1877?] 


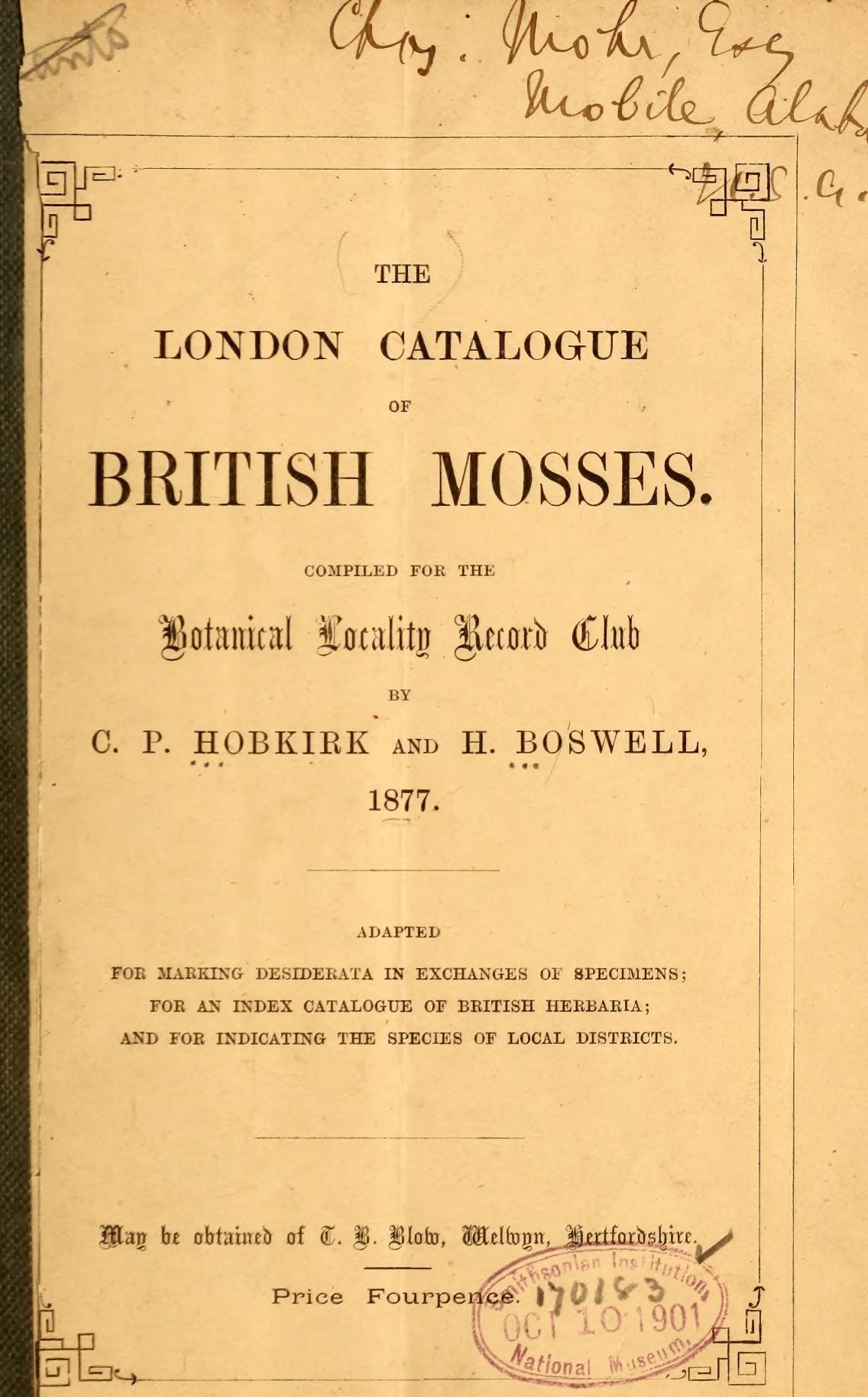





\section{THE LONDON CATALOGUE}

\section{(1) \\ BRITISH MOSSES}

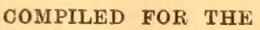

3

BY

C. P. HOBKIRK AND H. BOSWELL.

N.B.-Doubtful British species are placed in the General List in parentheses, with the sign *.

\section{SECTION I.-ACROCARPI.}

Tribe 1. SPHAGNACEA. Sphagnum, Dill.

1 acutifolium, Ehr.

$\beta$ deflexum.

$\gamma$ lilacinum.

$\delta$ purpureum.

$\varepsilon$ fuscum.

$\zeta$ luridum.

$\eta$ patulum.

$\imath$ arctum.

2 fimbriatum, Wils.

3 strictum, Lind.

(Girgensohni, Russ.)

4 squarrosum, Pers.

5 teres, Angst.

6 Lindbergii, Schpr.

7 intermedium, Hoffin.

$\beta$ riparium.

8 cuspidatum, Ehrh.

$\beta$ plumosum.

9 molle, Sull.

$\beta$ Muelleri, Schimp.

10 rigidum, Schpr.

$\beta$ compactum.
11 subsecundum, Nees.

$\beta$ contortum.

$\gamma$ turgidum.

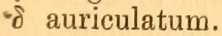

12 laricinum, Spruce.

(neglectum, Angst.)

13 rubellum, Wils.

14 tenellum, Ehrh.

(molluscum, Bruch.)

15 Austini, Sull.

16 papillosum, Lind.

$\beta$ confertum.

$\gamma$ stenophyllum.

17 cymbifolium, Ehrh.

$\beta$ squarrosulum.

$\gamma$ compactum.

(congestum.)

Tribe 2. ANDRE无ACE㘴.

Andreæa, Ehrh.

18 petrophila, Ehrh.

(rupestris, Hedw.)

19 alpestris, Schpr.

20 obovata, Theden.

21 alpina, Turn. 
22 rupestris, $L$. (Rothii, W. \& M.)

23 Grimsulana, Bruch.

24 crassinervia, Bruch.

25 falcata, Schpr.

26 nivalis, Hook.

Tribe 3. WEISSIACEA.

Systegium, Schpr.

(Phascum.)

27 crispum, Hedw.

28 Mittenii, Schpr.

29 multicapsulare, Sm.

Gymnostomum.

30 rostellatum, Brid. (Phascum -.)

31 tenue, Schrad.

32 rupestre, Schwg.

33 curvirostrum, Ehrh.

34 commutatum, Mitt.

35 microstomum, Hedw.

36 squarrosum, $N$. $\& H$.

37 tortile, Schwg.

Anœctangium, Schwg.

38 compactum, Schl.

$\beta$ pellucidum, Wils.

Weissia, Hedw.

39 viridula, Brid. (controversa.)

40 mucronata, Bruch.

41 crispula, Hedw.

42 cirrhata, Hedw.

Rhabdoweissia, B. \& S.

43 fugax, Hedw.

44 denticulata, Brid.

Cynodontium, $B . \& S$.

45 Bruntoni, B. \& $S$.

46 polycarpum, Ehrh.

(Didymodon Jenneri.)

47 strumiferum, Brid.

48 virens, Hedw.

Dichodontium, Schpr.

(Dicranum.)

49 pellucidum, $L$.

$\gamma$ serratum.

Dicranella, Schpr.

(Dicranum.)
50 crispa, Hedw.

51 Grevilleana, B. \& $S$.

52 Schreberi, Hedw.

$\beta$ lenta, Wils.

53 squarrosa, Schrad.

54 cerviculata, Hedw.

55 varia, $H e d w$.

$\beta$ fallax, Wils.

56 rufescens, Turn.

57 subulata, Hedw.

-58 curvata, Hedw.

59 heteromalla, Hedw.

$\delta$ sericea.

(Dicranodontium.)

Dicranum, Hed.

60 fulvellum, $S m$.

(Arctoa.)

61 Starkii, $W . \& M$.

62 falcatum, Hedw.

63 Blyttii, B. \& S.

64 montanum, Hedw.

65 flagellare, Hedw.

66 viride, $S m$.

67 Scottianum, Turn.

68 longifolium, Hedw.

69 arcticum, Schp. (glaciale.)

70 fuscescens, Turn.

71 scoparium, $L$.

72 majus, Turn.

73 palustre, Brid. (Bonjeanii.)

74 Schraderi, Schwg.

75 spurium, Hedw.

Dicranodontium, B. \& S.

76 longirostre, $W . \& M$.

77 aristatum, Schpr.

78 circinnatum, Wils.

Campylopus, Brid.

79 atro-virens, De Not.

(longipilus, p.p.)

80 brevipilus, $B$. \& $S$.

81 introflexus, Brid. (longipilus, p.p.) (polytrichoides, De Not.)

82 Shawii, Wils. 
83 alpinus, Schpr.

84 flexuosus, Brid.

85 (paradoxus, Wils.)

86 setifolius, Wils.

87 Schwarzii, Schp.

88 fragilis, $B . \& S$. $\beta$ densus.

89 Schimperi, Milde.

90 pyriformis, Brid. (turfaceus.) $\beta$ Mülleri.

91 brevifolius, Schpr.

Tribe 4. BRUCHIACEÆ.

Archidium, Brid.

92 phascoides, Brid.

Pleuridium, Brid.

(Phascum.)

93 nitidum, Hedw. $\beta$ strictum.

94 subulatum, $L$.

95 alternifolium, $B . \& S$.

Tribe 5. LEUCOBRYACE王.

Leucobryum, Hampe.

96 glaucum, $L$.

Tribe 6. SELIGERIACE E.

Seligeria, $B . \& S$.

97 Doniana, Sm.

(Anodus.)

98 pusilla, Hedw.

$\beta$ acutifolia.

99 calcarea, Dicks.

100 tristicha, Brid.

101 recurvata, Hedw.

102 subcernua, Schpr.

Campylostelium, B. \& S.

103 saxicola, $W . \& M$.

Brachyodus, Nees \& $H$.

104 trichodes, $W . \& M$.

Stylostegium, B. \& S.

105 cæspiticium, Schwg.

Blindia, B. \& S.

106 acuta, Hedw.

$\beta$ trichodes.
Tribe 7. POTTIACE无.

Ephemerella, Müll.

107 recurvifolia, Dicks.

(Phascum.)

Microbryum, Schp.

(Phascum.)

108 Floerkeanum, $W . \& M$.

Sphærangium, Schpr.

(Phascum.)

109 muticum, Schreb.

110 triquetrum, Spruce.

Phascum, $L$.

111 cuspidatum, Schreb.

112 bryoides, Dicks.

113 curvicollum, Hedw.

114 rectum, Sm.

Pottia, Ehr.

115 minutula, Schwg.

116 truncata, $L$.

117 intermedia, Turn.

118 Wilsoni, Hook.

119 crinita, Wils.

120 Heimii, Hedw.

121 Starkeana, Hedw. (Anacalypta.)

122 cæspitosa, Bruch. (Anacalypta.)

123 lanceolata, Dicks. (Anacalypta.)

124 latifolia, Schwg. (Anacalypta.)

125 viridifolia, Mitt.

126 littoralis, Mitt.

127 asperula, Mitt.

Didymodon, Hedw.

128 rubellus, $B$. \& $S$.

129 luridus, Hornsch.

130 flexifolius, Dicks.

$\beta$ gemmescens, Mitt.

131 cylindricus, Bruch.

132 sinuosus, Wils.

(Tortula.)

133 recurvifolius, Tayl.

Eucladium, B. \& S.

(Weissia.)

134 verticillatum, $L$, 
Ditrichum, Timm.

(Leptotrichum, Hampe.)

135 tortile, Schrad.

136 homomallum, Hedw.

137 flexicaule, Schwg.

138 subulatum, Bruch.

139 glaucescens, Hedw.

140 (vaginans, Sull.) $\beta$ glaciale.

Trichostomum, Sm.

141 tophaceum, Brid.

142 mutabile, Bruch.

143 crispulum, Bruch.

144 flavo-virens, Bruch.

145 nitidum, Lindb. (Tortula.)

146 littorale, Mitt. Barbula, Hedw. (Tortula.)

147 brevirostris, $B . \& S$

148 rigida, Schultz.

149 ambigua, $B . \& S$.

150 aloides, Koch.

15] cavifolia, Schpr.

152 lamellata, Lindb.

(B. concava, Schp.)

153 atro-virens, $S m$.

(Didymodon nervosus.)

154 cuneifolia, Dicks.

155 Vahliana, Schultz.

156 marginata, $B . \& S$.

157 canescens, Bruch.

158 muralis, $L$.

159 unguiculata, Dill.

160 fallax, Hedw.

161 recurvifolia, Schp.

162 rufa, Funk.

162 $a$ Woodii, Schp.

163 rigidula, Dicks. (Didymodon.)

164 spadicea, Mitt. (Trichost. rigidulum.)

165 cylindrica, Tayl. (insulana, De Not.)

166 vinealis, Brid.

167 Hornschuchiana, Schultz.

168 revoluta, Schwg.
169 convoluta, Hedw.

170 inclinata, Schwg.

171 tortuosa, $L$.

172 Hibernica, Mitt. (Anactang. Hornschuchianum.)

173 fragilis, Hook.

174 squarrosa, Brid.

175 Brebissoni, Brid.

(Cinclidotus riparius.)

176 subulata, $L$.

177 lævipila, Brid.

178 latifolia, B. \& S.

179 ruralis, $L$.

180 intermedia, Brid.

181 papillosa, Wils.

182 princeps, De Not. (Mülleri.)

Geheebia, Muell.

183 cataractarum, Schp. (Tortula gigantea.)

Trichodon, Schpr.

184 cylindricus, Hedw.

Ceratodon, Brid.

185 purpureus, $L$.

Distichium, B. \& S.

186 capillaceum, $L$,

187 inclinatum, Hedw.

Tribe 8. CALYMPERACE E.

Encalypta, Schreb.

188 commutata, Nees.

189 vulgaris, Hedw.

190 rhabdocarpa, Schwg.

191 ciliata, Hedw.

192 streptocarpa, $\mathrm{Hedw}$.

Tribe 9. GRIMMIACE $\nexists$.

Grimmia, Ehrh.

$\S 1$. Schistidium.

193 conferta, Funk.

$\beta$ pruinosa, Wils.

194 apocarpa, $L$,

195 maritima, Turn.

§ 2. Gastero-grimmia.

196 anodon, $B . \& S$. 
197 crinita, Brid.

§ 3. Grimmia.

198 orbicularis, B. \& $S$.

199 pulvinata, Dill.

200 Schultzii, Brid.

201 contorta, Wahl.

202 torquata, Grev.

203 funalis, Schwg. (spiralis.)

204 Mühlenbeckii, Schpr.

205 subsquarrosa, Wils.

206 trichophylla, Grev.

207 Hartmanni, Schpr.

208 elatior, Schpr. §4. Guembelia.

209 Donniana, Sm.

210 ovata, $W . \& M$.

211 leucophea, Grev.

212 commutata, Hueb.

213 montana, B. \& $S$.

214 Ungeri, Juratz.

215 elongata, Kaulf.

216 unicolor, Grev.

217 atrata, Miel.

218 Stirtoni, Schpr.

Racomitrium, Brid.

219 patens, Dicks.

220 ellipticum, Turn.

221 aciculare, $L$.

222 protensum, A. Braun.

223 sudeticum, Funk.

224 heterostichum, Hedw.

225 fasciculare, Schrad.

226 microcarpum, $H e d w$.

227 lanuginosum, Hedw.

228 canescens, Hedw. $\gamma$ ericoides.

Glyphomitrium, Brid. 229 Daviesii, Sm.

Ptychomitrium, B.\& S. 230 polyphyllum, Dicks.

Amphoridium, Schp. (Zygodon.)

231 lapponicum, Hedw. 232 Mougeotii, B. \& S.
Zygodon, $H . \& T$.

233 viridissimus, Dicks.

$\beta$ rupestris.

234 conoideus, Dicks.

235 Nowellii, Schp. (gracilis, Wils.)

236 Forsteri, Dicks.

Ulota, Mohr.

(Orthotrichum.)

237 Drummondii, Grev.

238 Ludwigii, Brid.

239 Bruchii, Hornsch.

240 calvescens, Wils.

241 crispa, Hedw.

242 intermedia, Schpr.

243 crispula, Bruch.

244 phyllantha, Brid.

245 Hutchinsiæ, Sm.

Orthotrichum, Hedw.

246 anomalum, $B$. \& S.

247 saxatile, Brid.

(anomalum, Bry. Brit.)

248 cupulatum, Hoffm.

$\beta$ nudum.

249 Sturmii, Hoppe.

250 Shawii, Wils. (orneum olim.)

251 rupestre, Schleich.

252 obtusifolium, Schrad.

253 affine, Schrad.

254 fastigiatum, Bruch.

255 speciosum, Nees.

256 patens, Bruch.

257 stramineum, Hornsch.

258 fallax, Swartz. (pumilum, Bry. Eur.)

259 pumilum, Swartz.

( fallax, Bry. Eur.)

260 tenellum, Bruch.

261 pallens, Bruch.

262 diaphanum, Schrad.

263 pulchellum, $\mathrm{Sm}$.

264 Lyellii, $H$. \& $T$.

265 leiocarpum, $B . \& S$.

266 Sprucei, Mont.

267 rivulare, Turn. 
Tribe 10. SPLACHNACE $\approx$.

Edipodium, Schwg. 268 Griffithianum, Dicks.

(Splachnobryum, Muell.) $269 *$ (Wrightii), Muell.

\section{Dissodon, Grev.}

270 splachnoides, Schwg.

Tayloria, Hook.

$271 *($ serrata, $H e d w$.)

272 tenuis, Dicks.

Tetraplodon, $B . \& S$.

273 angustatus, L. fll.; Hedw. 274 mnioides, L. fil.; Hedw.

Splachnum, $L$.

275 sphæricum, L. fil.

276 vasculosum, $L$.

277 ampullaceum, $L$.

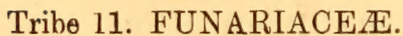

Discelium, Brid.

278 nudum, Dicks.

Ephemerum, Hampe.

(Phascum.)

279 serratum, Schreb.

280 tenerum, B. \& $S$.

281 cohærens, Hedw.

282 stenophyllum, Voit. $\beta$ brevifolium.

Physcomitrella, Schp.

(Phascum.) 283 patens, Hedw.

Physcomitrium, Brid. 284 sphæricum, Schwg. 285 pyriforme, $L$.

Entosthodon, Schwg. 286 ericetorum, Bals. 287 Templetoni, Hook.

Funaria, Schreb.

288 fascicularis, Dicks.

289 calcarea, Wahl.

290 hygrometrica, $L$.

291 microstoma, B. \& S.

Tribe 12. BARTRAMIACE $\mathrm{E}$. Amblyodon, P. Beauv. 292 dealbatus, Dicks.
Meesia, Hedw.

293 uliginosa, Hedw. $294 *$ (longiseta), Hedw.

Paludella, Ehrh. 295 squarrosa, $L$.

Catoscopium, Brid. 296 nigritum, Hedw.

Bartramia, Hedw. 297 stricta, Brid. 298 ithyphylla, Brid. 299 pomiformis, $L$. $\beta$ crispa.

300 Halleriana, Hedw. 301 Ederi, Gunn.

Conostomum, Swartz. 302 boreale, Swartz.

Philonotis, Brid.

(Bartramia.)

303 Wilsoni, $B$. \& $S$.

304 rigida, Brid.

305 parvula, Lindb.

306 (Marchica, Wild.)

307 fontana, $L$.

$\beta$ cæspitosa, Wils.

308 serrata, Mitt.

309 calcarea, $B . \& S$.

Breutelia, Schpr. (Bartramia.)

310 arcuata, Dicks.

Tribe 13. BRYACE $\$$.

Mielichhoferia, Hornsch. 311 nitida, Funk. $\delta$ elongata.

Orthodontium, Schwg. 312 gracile, Wils.

Leptobryum, Schpr. 313 pyriforme, $L$.

Webera, Hedw.

$$
\text { (Bryum, Dill.) }
$$

314 acuminata, Hoppe.

315 polymorpha, Hoppe

316 elongata, Dicks.

317 nutans, Schreb.

318 cruda, Schreb.

319 annotina, Hedw. 
320 Ludwigii, Spreng. (Breidleri, Jur.) $\gamma$ elata.

321 carnea, $L$.

322 albicans, Wahl.

Zieria, Schp.

(Bryum.)

323 julacea, Schpr. (B. Zierii.)

324 demissa, Hornsch.

Bryum, Dill.

325 pendulum, Hornsch. (cernuum.)

326 inclinatum, Swartz.

327 Warneum, Bland.

328 lacustre, Brid.

329 Barnesi, Wood; Schpr.

330 Marrattii, Wils.

331 calophyllum, $R$. Br.

332 uliginosum, Bruch.

333 intermedium, $W$. \& $M$.

334 bimum, Schreb.

335 torquescens, $B . \& S$.

336 pallescens, Schleich.

337 Sauteri, $B . \& S$.

338 erythrocarpum, Schwg. (sanguineum.)

339 murale, Wils.

(rubens, Mitt.?)

340 atro-purpureum, $W . \& M$.

341 alpinum, $L$.

342 Mühlenbeckii, $B . \& S$.

343 cæspiticium, $L$.

344 Funkii, Schwg.

345 argenteum, $L$.

346 capillare, $L$.

347 obconicum, Hornsch.

348 Donnianum, Grev.

349 canariense, Brid. (Billarderii.)

350 pallens, Swartz.

351 Stirtoni, Schp. (barbatum, Wils.)

352 Duvalii, Voit.

353 pseudo-triquetrum, Hed.

354 neodamense, Itzig.
355 turbinatum, Hedw.

356 (Schleicheri, Schw.)

$\gamma$ latifolium.

357 roseum, Schreb.

358 filiforme, Dicks.

(julaceum.)

359 catenulatum, Schpr.

Tribe 14. MNIACE $\mathrm{E}$.

Cinclidium, Swartz.

360 stygium, Swartz.

Mnium, $L$.

361 cuspidatum, Hedw.

362 affine, Bland.

363 undulatum, Hedw.

364 rostratum, Schrad.

365 hornum. L.

366 serratum, Schrad.

367 orthorrhynchum, B. \& $S$.

368 riparium, Mitt.

369 spinosum, Voit.

370 stellare, Hedw.

371 cinclidioides, Blytt.

372 punctatum, Hedw.

373 subglobosum, B. \& $S$.

Aulacomnium, Schwg.

374 androgynum, $L$.

375 palustre, $L$.

Timmia, Hedw.

376 austriaca, Hedw.

377 megapolitana, Hedw.

Tribe 15. GEORGIACEA.

Tetraphis, Hedw.

378 pellucida, $L$.

Tetradontium, Schwg. 379 Brownianum, Dicks.

Tribe 16. POLYTRICHACE 瓜. Oligotrichum, De Cand. 380 hercynicum, Ehrh.

Atrichum, P. Beauv.

381 undulatum, $L$.

382 angustatum, Brid.

383 tenellum, Rähl. 
384 crispum, James. (laxifolium, Wils.)

Pogonatum, P. Beauv.

385 nanum, Neck.

386 aloides, Hedw.

387 urnigerum, $L$.

388 alpinum, $L$.

Polytrichum, $L$.

389 sexangulare, Flörke.

390 gracile, Menz.

391 formosum, Hedw.
392 piliferum, Schreb.

393 juniperinum, Hedw.

394 strictum, Banks.

395 commune, $L$.

Diphyscium, Mohr.

396 foliosum, $L$.

Tribe 17. BUXBAUMIACE王.

Buxbaumia, Haller.

397 aphylla, Hall.

398 indusiata, Brid.

\section{SECTION II.-AMPHOCARPI.}

Tribe 18. FISSIDENTACE无.

Fissidens, $H e d w$.

399 bryoides, $H e d w$.

400 exilis, Hedw.

401 incurvus, Schwg.

402 viridulus, Wils.

403 tamarindifolius, Brid.

404 pusillus, Wils.

405 crassipes, Wils.

406 inconstans, Schpr.

407 osmundioides, Hedw.

408 decipiens, De Not.
409 adiantoides, Hedw.

410 taxifolius, $L$.

411 polyphyllus, Wils.

Tr.19. SCHISTOSTEGACE Schistostega, Mohr.

412 osmundacea, Dicks.

Tr. 20. EPIPTERYGIACE E. Epipterygium, Lindb.

413 Tozeri, Grev. (Bryum.)

\section{SeOtion III.-CLADOCARPI.}

Tribe 21. RIPARIACE无.

Cinclidotus, $P$. Beauv. 414 fontinaloides, Hedw.

Fontinalis, Dill.

415 antipyretica, $L$.

416 squamosa, $L$.

Dichelyma, Myrin.

$417 *$ (capillaceum), Dicks.
Tribe 22. CRYPH EACEA. Hedwigia, Ehrh.

418 ciliata, Dicks.

Hedwigidium, B. \& $S$.

419 imberbe, $S m$.

Cryphæa, Mohr.

420 heteromalla, Hedw.

\section{SECTION IV.-PLEUROCARPI.}

Tr. 23. LEUCODONTACE无. Myurium, Schpr.

Leptodon, Mohr. 421 Smithii, Dicks.

Loucodon, Schwg. 422 sciuroides, $L$. $\beta$ morensis.
423 Hebridarum, Schpr. (=L.Lagurus, $\beta$, Wils.)

Antitrichia, Brid. 424 curtipendula, $L$. 
Tribe 24. NECKERACE王.

Neckera, Hedw.

425 pennata, Hall.

426 pumila, Hedw.

$\beta$ Philippeana, Schpr.

427 crispa, $L$.

428 complanata, $L$.

Homalia, Brid.

429 trichomanoides, Schreb.

Tribe 25. HOOKERIACE

Daltonia, H. \& $T$. 430 splachnoides, $\mathrm{Sm}$.

Hookeria, $\mathrm{Sm}$.

431 læte-virens, $H . \& T$.

Pterygophyllum, Brid.

432 lucens, $S m$.

Tribe 26. FABRONIACE王.

Habrodon, Schpr.

433 Notarisii, Schpr.

(perpusillus, De Not.)

Myrinia, Schpr.

434 pulvinata, Wahl. (Leskea.)

Tribe 27. LESKEACEE.

Myurella, Schpr.

435 julacea, Villars.

(Leskea moniliformis, W.)

436 apiculata, Hueb.

Leskea, Hedw.

437 polycarpa, Ehrh.

438 nervosa, Schwg.

Anomodon, Hook.

439 longifolius, Schleich.

440 viticulosus, $L$.

Pseudo-leskea, B. \& S.

441 atro-virens, Dicks.

443 catenulata, Brid.

Heterocladium, Schpr.

$$
\text { (Hypmum.) }
$$

443 dimorphum, Brid.

444 heteropterum, Bruch.

Thuidium, Schpr.

$$
\text { (Hyp.) }
$$

$445 *$ (decipiens, De Not.)

446 tamariscinum, Hedv.

447 recognitum, Hedw. (delicatulum, L.)

448 abietinum, $L$.

449 Blandovii, $W . \& M$.

Tribe 28. HYPNACE $\mathrm{E}$.

Pterigynandrum, Hedw.

450 filiforme, Timm.

$\beta$ heteropterum, Brid.

Pterogonium, Swartz.

451 gracile, Dill.

Cylindrothecium, Schpr.

452 concinnum, $\mathrm{De}$ Not. (Montagnei.)

Thamnium, Schp.

453 alopecurum, $L$.

Climacium, Brid.

454 dendroides, $L$.

Pylaisia, Brid.

455 polyantha, Schreb.

(Leskea.)

Isothecium, Brid.

456 myurum, Poll.

Orthothecium, Schpr.

457 intricatum, Hartm.

(Leskea sub-rufa, Wils.)

458 rufescens, Dicks.

Homalothecium, Schpr.

459 sericeum, $L$.

Camptothecium, Schpr. (Hyp.)

460 lutescens, Huds.

461 nitens, Schreb.

Scleropodium, Schp.

(Hyp.)

462 cæspitosum, Wils.

463 illecebrum, Schwg.

Brachythecium, Schpr.

464 plicatum, Schl.

465 salebrosum, Hoffm.

$\gamma$ palustre.

(B. Mildeanum.)

466 glareosum, $B . \& S$.

467 cirrhosum, Schwg.

468 albicans, Neck. 
469 velutinum, $L$.

470 reflexum, $W . \& M$.

471 Starkii, Brid.

472 glaciale, $B . \& S$.

473 rutabulum, $L$.

474 campestre, $B . \& S$.

475 rivulare, $B . \& S$.

476 populeum, Hedw.

477 plumosum, Swartz.

Eurhynchium, Schpr.

478 myosuroides, $L$.

479 strigosum, Hoffm.

480 circinnatum, Brid.

481 striatulum, Spruce.

482 striatum, Schreb.

483 crassinervium, Tayl.

484 piliferum, Schreb.

485 speciosum, Brid.

486 hians, Hedw.

487 Swartzii, Turn.

(pralongum, Bry. Eur. et Schp. Syn.)

488 prælongum, Dill.

(Stokesii, Bry. Eur. \& Sch. Syn.)

$\beta$ Stokesii, Turn.

489 pumilum, Wils.

490) Teesdalii, Sm.

$\beta$ rigidulum.

Hyocomium, Schpr.

491 flagellare, Dicks.

Rhynchostegium, Schpr.

492 tenellum, Dicks.

493 depressum, Bruch.

494 confertum, Dicks.

495 megapolitanum, Bland.

496 murale, $H e d w$.

497 rusciforme, Weis.

Isopoterygium, Lindb. 498 demissum, Wils.

(Hурпит.)

Plagiothecium, Schpr.

499 latebricola, Wils. (Leskea.)

500 pulchellum, Hedw. 501 nitidulum, Wahl.
502 denticulatum, $L$.

$\beta$ obtusifolium.

$\gamma$ succulentum.

503 elegans, Hook.

504 sylvaticum, $L$.

505 undulatum, $L$.

506 Mühlenbeckii, Schpr.

507 silesiacum, Seliger.

Amblystegium, Schpr.

508 Sprucei, Bruch.

509 confervoides, Brid.

510 serpens, $L$.

511 radicale, $P$. Beauv. (varium, Auct.)

512 irriguum, Wils.

513 fluviatile, Swartz.

514 riparium, $L$.

Hypnum, Dill.

$\S 1$. Harpidium.

515 aduncum, Hedw. (Kneiffi, Bry. Brit.)

516 exannulatum, Gümb. (aduncum, Bry. Brit.)

$\beta$ stenophyllum, Wils.

517 vernicosum, $L i n d b$.

518 Cossoni, Schpr.

(intermedium, Lindb.?)

519 Sendtneri, Schp.

$\beta$ Wilsoni.

520 lycopodioides, Schwg.

521 revolvens, Swartz.

522 fluitans, $L$.

523 uncinatum, Hedw.

§ 2. Cratoneuron.

524 filicinum, $L$.

525 commutatum, Hedw.

526 falcatum, Brid.

527 sulcatum, Schpr.

$\beta$ subsulcatum.

$\S 3$. Rhytidium.

528 rugosum, Ehr.

$\S 4$. Homomalimum.

529 incurvatum, Brid. $\S 5$. Drepanium.

530 canariense, Mitt.

531 hamulosum, $B . \& S$. 
532 callichroum, Brid.

533 Bambergeri, Schp.

534 imponens, $\mathrm{Hed}$.

535 cupressiforme, $L$. $h$ resupinatum.

536 arcuatum, Lindb. (pratense, Bry. Brit.)

$537 *($ pratense, Koch. $)$ §6. Ctenidium.

538 molluscum, Hed. $\S 7$. Cteniun.

539 crista-castrensis, $L$. § 8. Liminobium.

540 palustre, $L$.

541 molle, Dicks.

542 dilatatum, Wils.

543 eugyrium, Schp.

544 arcticum, Somm.

545 ochraceum, Turn. §. Chrysobryum.

546 micans, Wils. § 10. CAMPYLIOM.

547 Halleri, $L$.

548 Sommerfelti, Myr.
549 elodes, Spruce.

550 chrysophyllum, Brid.

551 polygamum, $B . \& S$.

552 stellatum, Schreb. §11. Calliergon.

553 cordifolium, Hedw.

554 giganteum, $S c h p$.

555 sarmentosum, Wahl.

556 cuspidatum, $L$.

557 Schreberi, Ehrh.

558 purum, $L$.

559 stramineum, Dicks.

560 trifarium, $W$. \& $M$.

561 scorpioides, $L$.

Hylocomium, Schp.

562 splendens, Dill. Hedw.

563 umbratum, Schreb.

564 Oakesii, Sull.

565 brevirostre. Ehrh.

566 squarrosum, $L$. $\boldsymbol{\beta}$ calvescens, Wils.

567 loreum, $L$.

568 triquetrum, $L$. 



Pressboard

Pamphlet

Binder

Gaylord Bros., Inc. Makers

Syracuse, N. Y.

PAT, JAN. 21, 1908 
